\title{
Prosthetic valve endocarditis after transcatheter CoreValve Evolut R bioprosthesis implantation
}

\author{
Karol Zbroński, Zenon Huczek, Piotr Scisło, Janusz Kochman, Krzysztof J. Filipiak, Grzegorz Opolski
}

First Department of Cardiology, Medical University of Warsaw, Warsaw, Poland

Adv Interv Cardiol 2016; 12, 4 (46): 383-385

DOI: $10.5114 /$ aic.2016.63643

Prosthetic valve endocarditis (PVE) affects $1-6 \%$ of prosthetic valve recipients and in patients treated with transcatheter aortic valve implantation (TAVI) has an incidence of $0.3-2.3 \%$ per patient-year [1-3].

A 79-year-old man who underwent TAVI with a Medtronic Evolut R $29 \mathrm{~mm}$ bioprosthesis 7 months earlier was admitted due to recurring episodes of fever up to $39^{\circ} \mathrm{C}$ and mild worsening of exercise tolerance lasting for 4 months. Other relevant medical history consisted of chronic obstructive pulmonary disease (COPD) and penicillin allergy. During the past months the patient was hospitalized twice (once due to fever, the second time due to syncope), but the symptoms were attributed to COPD exacerbation.

At admission, physical examination was remarkable for systolic murmur over the mitral valve. No fever, chest pain, cough, or meningeal signs were appreciated. Electrocardiogram (ECG) demonstrated sinus rhythm with left bundle branch block observed in previous ECGs. Chest $X$-ray revealed an enlarged cardiac silhouette. Transthoracic echocardiography (TTE) demonstrated mild mitral regurgitation (Figure $1 \mathrm{~A}$ ) and $15 \mathrm{~mm}$ hyperechogenic structures on the mitral valve described as possible vegetations (Figure $1 \mathrm{~B}$ ). Laboratory findings revealed a white blood cell count of $19 \times 10^{3} / \mu \mathrm{l}$, N-terminal prohormone of brain natriuretic peptide of $1000 \mathrm{pg} / \mathrm{ml}$ and C-reactive protein of $123 \mathrm{mg} / \mathrm{l}$.

Due to high clinical suspicion of PVE, the patient was started on vancomycin, gentamicin and rifampin. Beforehand, three sets of blood cultures were collected, which were found to be positive for methicillin-sensitive Staphylococcus capitis. Transesophageal echocardiography (TEE) confirmed PVE demonstrating vegetations on the leaflet located in the vicinity of the native right coronary cusp (Figures $1 \mathrm{C}$ and D). Further treatment with rifampin and gradually increased doses of vancomycin (gentamicin was discontinued after 2 weeks due to transitory worsening of the renal function) led to clinical improvement, whilst laboratory examinations showed C-reactive protein and white blood cell (WBC) normalization. Even though repeated TEEs demonstrated persistence of vegetations, clinically the PVE was deemed healed. After 6 weeks antibiotics were discontinued, blood cultures were negative and the patient was discharged.

A recent study [2] on 7944 patients found two independent factors associated with infective endocarditis (IE) prevalence - orotracheal intubation and use of self-expandable CoreValves. Among factors possibly responsible for PVE prevalence in the cohort treated with CoreValves, the following were suggested: larger stent frame, larger contact surface area between tissues and the frame, and paravalvular leak (PVL).

In another study [4] risk factors for post-TAVI infective endocarditis development were male sex, low implantation position, implantation of $>1$ prosthesis, and vascular and bleeding complications.

The newly released Medtronic CoreValve Evolut R system shows promising features as compared to the first generation of CoreValves in terms of PVE prevention. Firstly, its height is $10 \%$ shorter, so both the stent frame and the contact surface between tissues and the frame are smaller. Secondly, it has an extended skirt designed to minimize PVL. Thirdly, the valve's repositionability facilitates optimal implantation and may reduce the need for valve-in-valve procedures. Finally, its smaller insertion profile could minimize occurrence of vascular and bleeding complications and promote TAVI without orotracheal intubation.

In the case of our patient, potential risk factors included male sex, use of a self-expandable valve and orotracheal intubation. On the other hand, IE prophylaxis was administered (cefazolin), only one valve was used, the position was optimal, and neither PVL nor vascular and bleeding complications were present.

Corresponding author:

Karol Zbroński MD, First Department of Cardiology, Medical University of Warsaw, 1a Banacha St, 02-097 Warsaw, Poland, phone: +48 607516 810, e-mail: karol.zbronski@gmail.com

Received: 30.07.2016, accepted: 6.10.2016. 

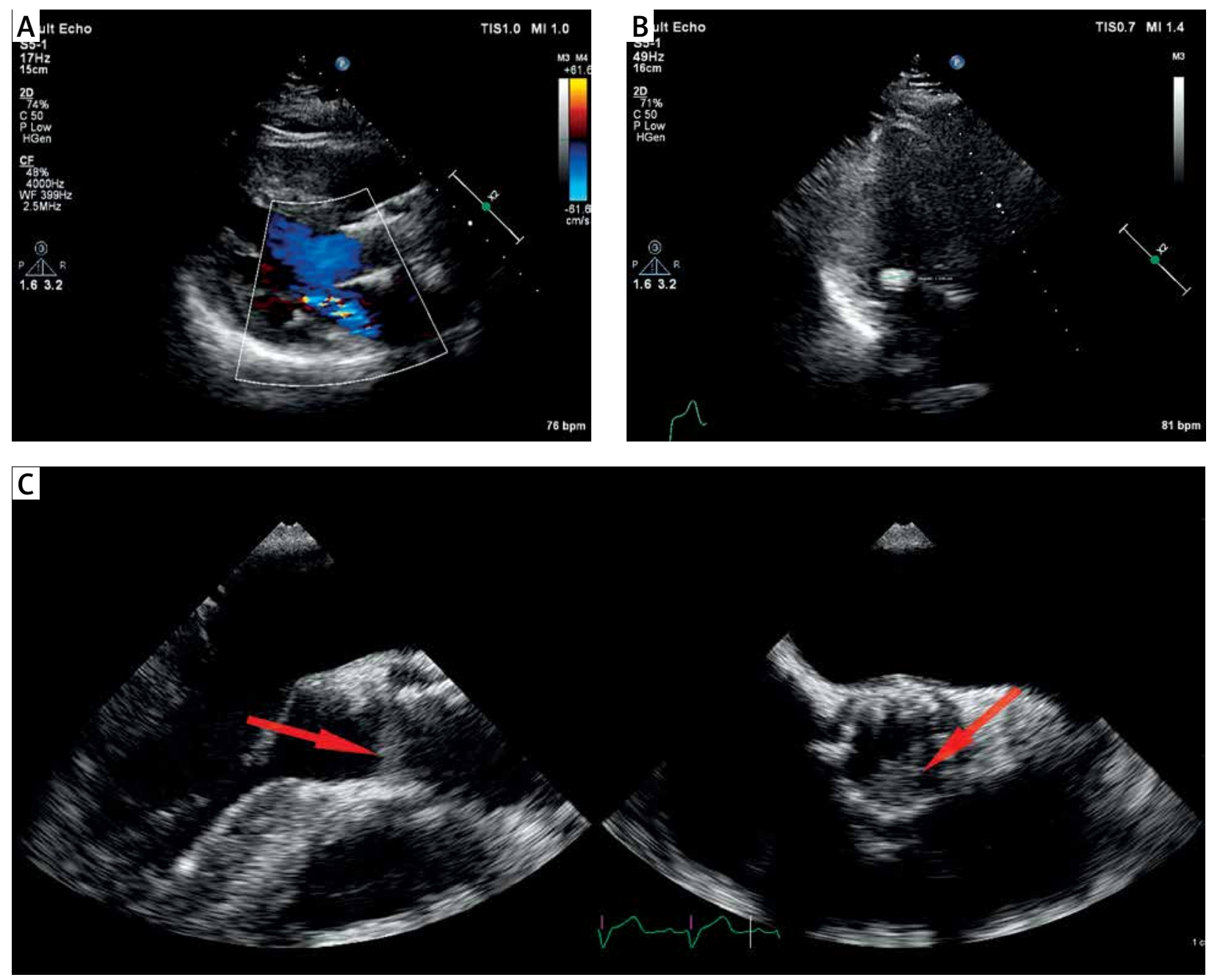

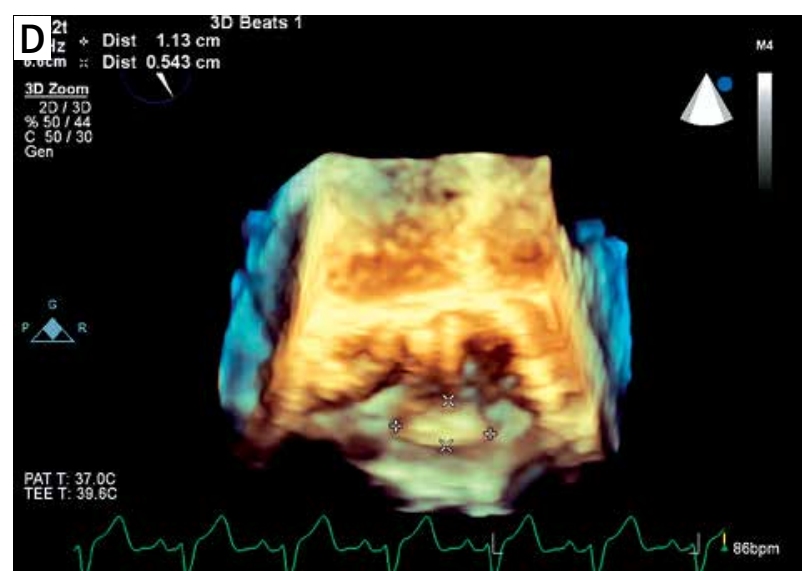

In conclusion, despite potentially protective modifications in design, PVE on the Evolut $R$ valve may still occur and in order to accurately assess PVE prevalence larger registry-based studies are required. Furthermore, one must emphasize the need for repeated TEEs and blood cultures as well as using new imaging modalities such as cardiac computed tomography (CT), positron
Figure 1. A - Transthoracic echocardiography with color Doppler, parasternal long-axis view. Mild mitral regurgitation is apparent. B - Transthoracic echocardiography, parasternal long-axis view. Hyperechogenic structures on the posterior leaflet of the mitral valve described as possible vegetations. $\mathbf{C}$ - Biplane transesophageal echocardiography, mid-esophageal aortic valve views. Large vegetations (arrows) are visible inside the bioprosthesis, on the leaflet located in the vicinity of the native right coronary cusp. D - Three-dimensional transesophageal echocardiography. Large vegetations (arrows) can be seen inside the bioprosthesis, on the leaflet located in the vicinity of the native right coronary cusp

emission tomography (PET)/CT and single-photon emission computed tomography (SPECT)/CT in cases with a high level of clinical IE suspicion, especially in prosthetic valve recipients.

\section{Conflict of interest}

The authors declare no conflict of interest. 


\section{References}

1. Habib G, Lancellotti P, Antunes MJ, et al. 2015 ESC Guidelines for the management of infective endocarditis: The Task Force for the Management of Infective Endocarditis of the European Society of Cardiology (ESC). Endorsed by: European Association for Cardio-Thoracic Surgery (EACTS), the European Association of Nuclear Medicine (EANM). Eur Heart J 2015; 36: 3075-128.

2. Amat-Santos IJ, Messika-Zeitoun D, Eltchaninoff $H$, et al. Infective endocarditis after transcatheter aortic valve implantation: results from a large multicenter registry. Circulation 2015; 131 : 1566-74.

3. Kochman J, Kołtowski L, Huczek Z, et al. Direct transcatheter aortic valve implantation - one-year outcome of a case control study. Postep Kardiol Interw 2014; 10: 250-7.

4. Olsen NT, De Backer O, Thyregod HG, et al. Prosthetic valve endocarditis after transcatheter aortic valve implantation. Circ Cardiovasc Interv 2015; 8: pii: e001939. 